\title{
Multidimensional knapsack problem optimization using a binary particle swarm model with genetic operations
}

\author{
Luis Fernando Mingo López Nuria Gómez Blas - Alberto Arteta Albert
}

\begin{abstract}
Particle swarm optimization is a heuristic and stochastic technique inspired by the flock of birds when looking for food. It is currently being used to solve continuous and discrete optimization problems. This paper proposes a hybrid, genetic inspired algorithm that uses random mutation/crossover operations and adds penalty functions to solve a particular case: the multidimensional knapsack problem. The algorithm implementation uses particle swarm for binary variables with a genetic operator. The particles update is performed in the following way: first using the iterative process (standard algorithm) described in the PSO algorithm and then using the best particle position (local) and the best global position to perform a random crossover/mutation with the original particle. The mutation and crossover operations specifically apply to personal and global best individuals. The obtained results are promising compared to those obtained by using the probability binary particle swarm optimization algorithm.
\end{abstract}

Keywords Binary particle swarm optimization . Combinatory optimization - Multidimensional knapsack problem · Genetic operations

\section{Introduction}

Combinatory optimization research deals with problems that have a finite number of outcomes (Korte and Vygen 2007). As part of the optimization process, the paper focuses on the solution that maximizes or minimizes specific fitness functions according to the following:

$\min / \max f(x), x \in \mathcal{S}$

where $\mathcal{S}$ is a finite set of possible solutions.

The cost function (fitness function) $f$ is an application from $\mathcal{S}$ into $\Re$

$f: \mathcal{S} \rightarrow \Re$

When the problem is minimization (or maximization), the solution consists of finding a value $s^{*}$ that fulfills:

$f\left(s^{*}\right)=\min _{s_{i} \in \mathcal{S}} f\left(s_{i}\right)$ or $f\left(s^{*}\right)=\max _{s_{i} \in \mathcal{S}} f\left(s_{i}\right)$

where $s^{*}$ is a global minimum.

In computational theory, a decision problem is a question in some formal system with a yes-or-no answer, depending on the values of some input parameters. Decision problems are typically defined in mathematical theory and computational complexity as decidable or non-decidable problems, that is to say, It is possible to find a method that determines the existence of set containing an instance of the problem; 
some of the most important problems in mathematics are undecidable yet.

The easiest solution for this kind of decision problems is to choose between two alternatives: either 0 or 1 . In this case, binary variables are used $x \in\{0,1\}$ to design the process resolution. However, some issues emerge when using a high numbers of binary variables as this kind of decision problems are more difficult to deal with. In the simplest version of a linear decision model, the evaluation of decision process is basically to find a linear combination of the decisions assigned values (Kellerer et al. 2004).

For example, this occurs in the Boolean Satisfiability problem (Zhang and Malik 2002), commonly referred as SAT. The solution to SAT is to find an interpretation that satisfies a given Boolean formula. More in deep, the problem requires to determine whether the variables of a given boolean formula can be consistently replaced by the values true or false. By doing so, the formula evaluates to true. If this is the case, the formula is called satisfiable. On the other hand, if no such values assignment is found, the function expressed by the formula is false and unsatisfiable.

The 0-1 Knapsack problem (KP) is another example that falls into this category (Martello and Toth 1985). This consists of placing a finite number of objects in a knapsack, in which each object has an associated value and volume; the solution not only should not exceed the capacity of the container but must also obtain the highest possible benefit.

This paper propose a hybrid algorithm, based on the metaheuristic particle swarm optimization (Kennedy and Eberhart 1995), that addresses problems in discrete models, such as the multidimensional and the multi restricted Knapsack problem. To show the robustness of the proposed algorithm in handling other problem types, in the further research, the algorithm must be tested and evaluated over other binary test problems. Moreover, statistical tests such as Friedman test must be employed to confirm the performance of the proposed methods statistically. Finally, testing against existing non-PSO state-of-the-art methods is helpful in establishing the algorithms for general purposes.

\section{Knapsack problem}

Knapsack problem (KP) is a combinatorial optimization problem. Given a set of items with weight and value, the problem consists of finding the number of items to be included in a knapsack so the total weight is less than or equal to a given threshold (knapsack capacity) and the total value (worth) is as large as possible. This is a common problem that a traveler faces when traveling with a fixed-size knapsack that must host the most valuable items. This kind of known problems often appear in fields such as combinatorics, com- puter science, complexity theory, cryptography and applied mathematics (Martello and Toth 1990).

The way to model it is as follows:

Every decision, $i$, is equal to 0 or 1 and the benefit value $p_{i}>0 . w_{i}$ represents the weight of the object. $\mathrm{A}$ set of decisions is feasible if the sum of all of them is not greater than the capacity $\mathcal{C}$. This restriction can be checked in Eq. (4).

$$
\sum_{i=1}^{n} w_{i} x_{i} \leq C
$$

The binary Knapsack problem solution consists of finding the $x_{i}$ values obtain the maximal benefit; see Eq. (5) (Kellerer et al. 2004).

$\max \sum_{i=1}^{n} p_{i} x_{i}$

where $\sum_{i=1}^{n} w_{i} x_{i} \leq C$ with $x_{i} \in\{0,1\}, i=1, \ldots, n$.

There are many other problems that are modeled in a similar way to the binary knapsack problem such as the financial portfolio optimization, dynamic job programming, employees hiring, logistic operations.

There are also different variations of KP such as the multidimensional knapsack problem (MKP), which has several restrictions and is more complicated to solve. This problem is currently classified as NP-hard, see Labed et al. (2011), Wan and Nolle (2009) and it is formulated as follows:

$\max \sum_{i=1}^{n} p_{i} x_{i}$

where $\sum_{i=1}^{n} w_{i j} x_{i} \leq C_{j}, j=1, \ldots, m$ with $x_{i} \in$ $\{0,1\}, i=1, \ldots, n$ and $\mathcal{C}_{j}>0, w_{i j} \geq 0, p_{i}>0$, $w_{i j}<C_{j} \leq \sum_{j=1}^{n} w_{i j}, \forall i, j$.

Both kinds of problems have been approached by using linear programming and stochastic algorithms. According to Wan and Nolle (2009), stochastic algorithms have used less computational resources than linear programming obtaining similar results. Furthermore, particle swarm optimization technique has been deployed in several optimization problems such as combinatory optimization, travel salesman problem (TSP) and flow shop problems, see Parsopoulos and Vrahatis (2002b).

\subsection{Multidimensional KP and PSO}

The $0-1$ multidimensional knapsack problem (MKP) is categorized as a difficult NP-hard combinatorial optimization problem. Some population search algorithms try to solve these problems. Specifically, the particle swarm optimiza- 
tion (PSO) model and its variants have proved to be a fairly good technique to deal with the MKP.

The essential particle swarm optimization queen (EPSOq) is one of the recent discrete PSO versions that further simplifies the PSO principles and improves its optimization ability. Hybridization is a principle in combining two (or more) approaches. By doing so, the resulting algorithm includes positive features of both (or all) the algorithms. Ktari and Chabchoub (2013) propose a new heuristic approach that is inspired from the Tabu Search and incorporated in the EPSOq algorithm in order to obtain an enhanced discrete PSO version. Experimentally, this approach is able to optimally choose a large-scale of strongly correlated binary values representing multidimensional knapsack problem (MKP) instances. Computational results show that TEPSOq has outperforms not only the EPSOq, but also other existing PSObased approaches and some other meta-heuristics in solving the $0-1$ MKP. Furthermore, this algorithm is able to find extremely closed solutions and even to reach the best known results available in the literature.

A more recent research (Haddar et al. 2016a) proposes a new hybrid heuristic approach that combines the quantum particle swarm optimization technique (Haddar et al. 2016b) with a local search method to solve the multidimensional knapsack problem. This approach also incorporates a heuristic repair operator that uses problem-specific knowledge instead of the standard penalty function technique commonly used for constrained problems. Experimental results obtained on a wide set of benchmark problems clearly demonstrate the competitiveness of the proposed method compared to the state-of-the-art heuristic methods.

Chin-Jung Lin proposes a novel PSO algorithm, the binary PSO based on surrogate information with proportional acceleration coefficients (BPSOSIPAC) (Lin et al. 2016). The particles movement of BPSOSIPAC just reuses the proportional acceleration coefficients from the traditional PSOs and incorporate cognition and social learning factors, which reduces the possibilities of influencing the particles movement and the time of computing the velocity of the particles at each iteration. Simultaneously, it stably drives the quality of the MKPs benchmark problems toward the optimal direction in every experiment. The simulation and evaluation results showed that BPSOSIPAC is superior to the other methods in any case for low-dimensional or high-dimensional knapsack problems. Thus, BPSOSIPAC is definitively a promising algorithm in solving multidimensional knapsack problems.

\section{Particle swarm optimization}

Particle swarm optimization (PSO) is a heuristic optimization technique introduced by Kennedy and Eberhart (1995). It is based on the evolution of an initial random set of points in a multidimensional space. This set is addressed as population. The population is moving over a searching space by an algorithm that looks for a solution. A particle is an element of the population and represents a candidate solution to the problem. Kennedy and Eberhart (1997) introduced a new algorithm with binary and discrete variables. This approach is inspired by flocks of birds when moving to obtain food. The initial population and the evolutionary process are generated in the same stochastic way as the one in evolutionary algorithms; every single individual of the population is a particle with a speed and location (multidimensional coordinates).

Particles are evolutionary solutions that move based on the combination of their best personal solution (pbest - personal best) and the best solution reached by their neighbor's particles (lbest-local best). When the neighborhood is designed following the standards of a star topology, the lbest term is referred as gbest (global best) since all particles are neighbors (see Fig. 2). Furthermore, the performance (fitness function) of every particle is computed in every evolutionary step.

In the binary problem, the particles are strings with 0 's and 1's so they can be used in a similar way than genetic algorithm individuals.

Initially, particles are randomly distributed in the problem domain, and they move along the searching space by using specific rules for each evolutionary step. The goal consists of obtaining the best solution (max or min fitness value) moving (evolving) every particle closer to the neighbor that has the best fitness so far; in that way, it influences the other particles too; in that way, the algorithm updates the swarm of particles in every evolutionary step by updating the position and velocity of every particle.

\subsection{Standard PSO model}

Let us define the following symbols to represent properties of a particle:

- $x_{i}$ is the current position of particle $i$

$-v_{i}$ is the current velocity of particle $i$

- $p_{\text {Best }}$ is the personal best position of the particle

- $g_{\text {Best }}$ is the global best particle

$-c_{1} \in R$ is the personal influence (acceleration coefficient)

$-c_{2} \in R$ is the global influence (acceleration coefficient)

- $r_{1}$ and $r_{2}$ are random numbers distributed using a uniform pattern on interval $[0,1]$.

With these notations, the formula to calculate a particle's velocity at time $t+1$ (time is simulated using iteration number) is:

$$
\begin{aligned}
v_{i}(t+1)= & v_{i}(t)+c_{1} * r_{1} *\left(p_{\text {Best }}-x_{i}\right) \\
& +c_{2} * r_{2} *\left(g_{\text {Best }}-x_{i}\right)
\end{aligned}
$$


Fig. 1 Particle swarm optimization model: position update using a star topology $\left(g_{\text {Best }}\right)$ and an inertia $w$ term

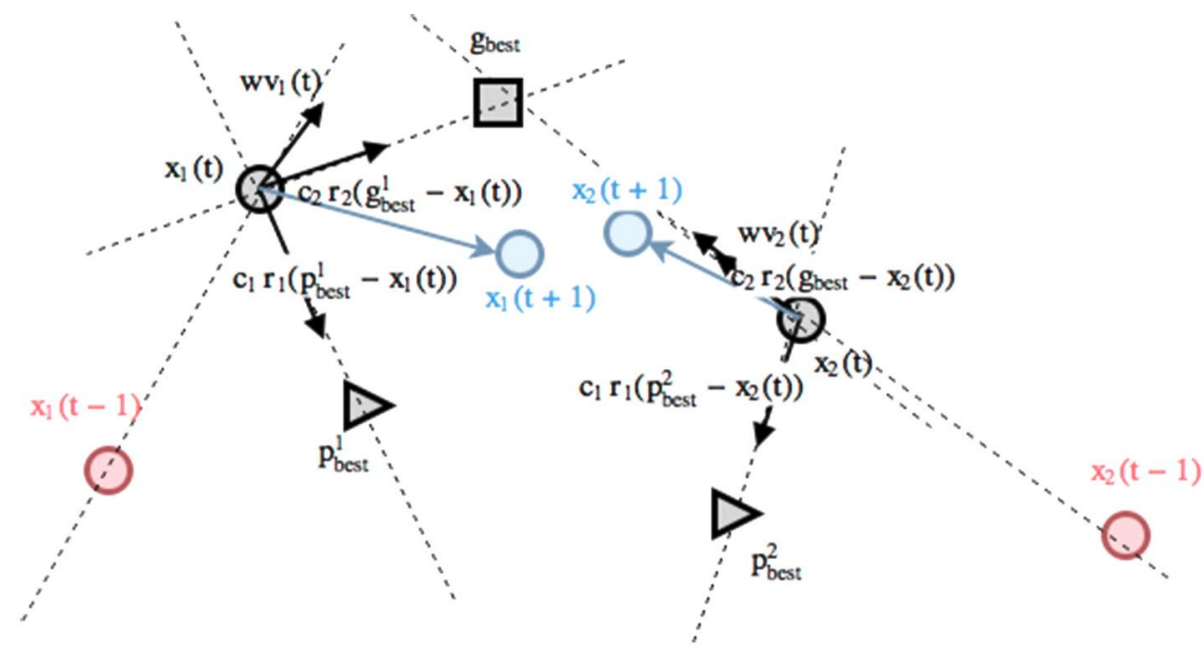

Fig. 2 Particle swarm topology: $g_{\text {Best }}$ (star) topology and $l_{\text {Best }}$ (ring) topology (from left to right)
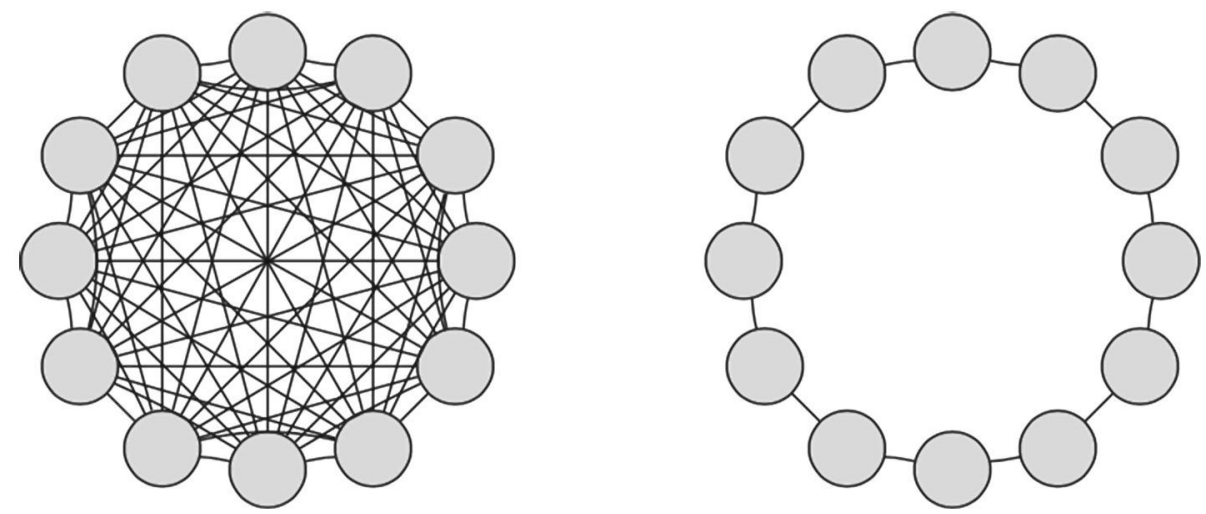

where, $r_{1}$ and $r_{2}$ are randomly generated for every velocity update and $0 \leq r_{1}, r_{2} \leq 1$. Such parameters should be set with a different value in each iteration. And $c_{1}, c_{2}$ are user-defined values called acceleration coefficients where $0 \leq c_{1}, c_{2} \leq 2$. Their value depends on the problem to be optimized.

New particle position at time $t+1$ just adds the newly calculated velocity to its current position at time $t$. In other words, the position now is the previous one adding its velocity, see Fig. 1.

$x_{i}(t+1)=x_{i}(t)+v_{i}(t+1)$

Note that $g_{\text {Best }}$ refers to a star topology, see Fig. 2 .

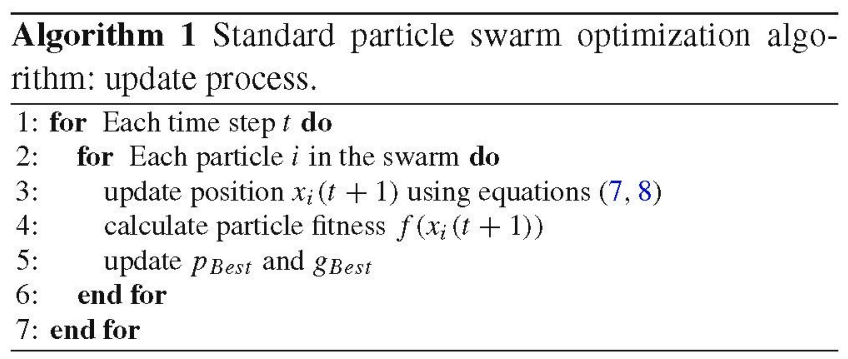

\subsubsection{Swarm topology}

$g_{\text {Best }}$ is used because the particles reside in a star topology neighborhood. This means that all particles are connected to each other, see Fig. 2. Another topology, the $l_{\text {Best }}$ swarm model, often referred to as a local topology, see Fig. 2 (right), constitutes perhaps the most significant variation in the original PSO algorithm, and was in fact proposed in one of the very first PSO publications (Eberhart and Kennedy 1995). For one reason or another, this topology has not been widely used in the community up to this point. Original investigations into this model using the original PSO algorithm showed inferior performance when compared to the global $g_{\text {Best }}$ model, or global topology, shown in Fig. 2 (left), but more recent research has revealed that $l_{\text {Best }}$ swarms return improved results across many standard problem sets when used in conjunction with other improvements to the algorithm (Kennedy and Mendes 2006). It is important to note that the local topology only affects Eq. (7), where:

$$
\begin{aligned}
v_{i}(t+1)= & v_{i}(t)+c_{1} * r_{1} *\left(p_{\text {Best }}-x_{i}\right) \\
& +c_{2} * r_{2} *\left(l_{\text {Best }}-x_{i}\right)
\end{aligned}
$$


Much of the PSO literature uses the term local topology to describe not just a single swarm model, but applies it to any swarm model without global communication. A number of different limited communication topologies have been tested with varying results; the $l_{\text {Best }}$ model shown is perhaps the simplest form of a local topology, what is known as the ring model, shown in Fig. 2 (right). The $l_{\text {Best }}$ ring model connects each particle to only two other particles in the swarm, in contrast to the $g_{\text {Best }}$ model where every particle is able to obtain information from the very best particle in the entire swarm population.

The advantage of the $l_{\text {Best }}$ model appears to lie in its slower convergence rate relative to the $g_{\text {Best }}$ model. Having convergence take place when the swarm has found the global optimum is obviously beneficial, but when the convergedupon location is suboptimal, it is referred to as premature and is undesirable as it prevents the algorithm from escaping from an inferior local optimum.

Ironically, it is the slower rate of convergence of the $l_{\text {Best }}$ model that is most responsible for the general disregard of it as an alternative up to this point. The much faster convergence of the $g_{\text {Best }}$ model seems to indicate that it produces superior performance, but this is misleading. While results for the $g_{\text {Best }}$ model are indeed superior for many problems relatively early in the optimization process, the best found fitness for the $l_{\text {Best }}$ model quite often surpasses that of the $g_{\text {Best }}$ after some number of function evaluations have been performed, particularly on multimodal problems.

Despite the advantages of a local topology, it is important to note that it should not always be considered to be the optimal choice in all situations. The faster convergence rate of a global topology will usually result in better performance on simple unimodal problems than that of any local topology due to the lack of any danger of convergence on a suboptimal local minima. Even on some very complex multimodal problems, a $g_{\text {Best }}$ model swarm can deliver performance competitive with the $l_{\text {Best }}$ model, given proper circumstances. For thorough, rigorous results, tests should be run using both topologies, but in the interest of having a single standard PSO algorithm, the superior performance of the $l_{\text {Best }}$ model over the majority of benchmarks qualifies it as the better choice for cases where a straightforward means of comparison is desired. In any case, modern research performed using only swarms with a global topology is incomplete at best (Eberhart and Kennedy 1995; Kennedy and Mendes 2006).

The inclusion of the local ring topology as part of a standard algorithm for particle swarm optimization comes with a caveat, however. Given the slower convergence of the $l_{\text {Best }}$ model, more function evaluations are required for the improved performance to be seen. This is especially important on unimodal functions, where the fast convergence of the $g_{\text {Best }}$ model combined with a single minima in the feasible search space results in quicker performance than that of the $g_{\text {Best }}$ swarm with its limited communication.

\subsection{Binary PSO model}

Kennedy and Eberhart (1997) proposed a discrete binary version of PSO for binary problems. In their model, a particle will decide on yes or no, also this binary values can be a representation of a real value in binary search space. The binary model is based on the probability of making decisions. Making a decision is modeled as 1 (yes) or 0 (no). In the end, a particle can decide yes or no ( 1 or 0$)$ based on its fitness value and some probability rules.

The probability $P$ of getting a 1 in particle $i$ in dimension $d$ at time $t$ (represented by $\left.x_{i d}(t)\right)$ depends on:

$P\left(x_{i d}(t+1)=1\right)=f\left(x_{i d}(t), v_{i d}(t), p_{i d}, p_{g d}\right)$

where:

- $x_{i d}(t)$ is the status of the particle $i$ in the position $d$

$-v_{i d}(t)$ is the velocity of the individual

- $p_{i d}$ is the best position of the particle $i$, found in the dimension $d$

- $p_{g d}$ is the best position of all its neighbors, with dimension $d$

In the binary PSO, the particles personal best and global best is updated as in continuous version. The major difference between binary PSO with continuous version is that velocities of the particles are rather defined in terms of probabilities that a bit will change to one. Using this definition a velocity must be restricted within the range $[0,1]$. So a map is introduced to map all real-valued numbers of velocity to the range $[0,1]$. New velocity $v_{i d}^{\prime}$ is computed as:

$v_{i d}^{\prime}(t)=\operatorname{sigmoid}\left(v_{i d}(t)\right)=\frac{1}{1+e^{-v_{i j}(t)}}$

In order to update $v_{i d}(t)$, the following formula is used:

$$
\begin{aligned}
v_{i d}(t+1)= & v_{i d}(t) \\
& +\varphi_{1} \epsilon_{1}\left(p_{i d}-x_{i d}(t)\right) \\
& +\varphi_{2} \epsilon_{2}\left(p_{g d}-x_{i d}(t)\right)
\end{aligned}
$$

where $\varphi_{1}$ y $\varphi_{2}$ represent cognitive and social factors fulfilling $\varphi_{1}+\varphi_{2}=4$ and $\left(\epsilon_{1}, \epsilon_{2}\right)$ are independent random uniform numbers within $(0,1)$.

The following equation updates the particle's position:

$$
x_{i d}(t)= \begin{cases}1 & \text { if } \rho_{i d}<s\left(v_{i d}(t)\right) \\ 0 & \text { otherwise }\end{cases}
$$


where $\rho_{i}$ is an array of random numbers and $s$ is a function that returns values within $[0,1]$, which makes the function act as a threshold [similar to the sigmoid function, see Eq. (11)], which is widely used in neural networks.

\subsection{Model variants}

Regarding the PSO algorithm, different variants have been developed, aimed at speeding up the convergence of it. In addition to the unconstrained optimization problem in discrete or continuous variable, the multitarget problem and the constrained problem have been addressed. The new guidelines are aimed at avoiding PSO stagnation of the local optimal solutions.

Shi and Eberhart (1998) proposed adjustments to the velocities of the particles by using a factor $w$ called inertial weight. This factor utilizes the inertia of the particles in the process of friction when they are moving. This modification in the algorithm is done to control the search space. In order to do that, it must change (14). The large inertia weight makes the global search easier; however, small inertia weight does not improve local search. That is why was the initial value is greater than 1.0 to promote global exploration and then gradually decreases to obtain more refined solutions. The algorithm decreases linearly at each iteration. Moreover, the use of inertial weight removes the restriction $V_{\max }$ on the velocity.

$v_{d}^{(i)}=w v_{d}^{(i)}+c_{1} \epsilon_{1}\left(p_{d}^{(i)}-x_{d}^{(i)}\right)+c_{2} \epsilon_{2}\left(g_{d}^{(i)}-x_{d}^{(i)}\right)$

In each iteration, inertia weight decrease linearly through the following expression:

$w=w_{\max }-\left(w_{\max }-w_{\min }\right) \frac{g}{G}$

$g$ is the index of the generation, $G$ is the maximum number of iterations previously determined, $w_{\max }$ is a value greater than 1 , and $w_{\min }$ a value under 0.5 . This variation of the method has proven to accelerate convergence.

Term velocity clamping has not been previously included, but it is necessary to restrain velocity updates. Without doing so, particles would move too far from the search space, ignoring the current solution. If the search space has a range $\left[-x_{\max }, x_{\max }\right]$, then the velocity should also have a range $\left[-v_{\max }, v_{\max }\right]$. Our proposal for $v_{\max }$ is $k * x_{\max }, k$ being the clamping factor between 0.1 and 1 . The maximum velocity works as a constraint to control the global exploration of a swarm. If the value is too high, particles might bypass good solution and the exploration would be poor. On the other hand, slow particles might only be searching locally which would be good for local solutions but not for finding a global one; this means that the exploiting process becomes poor too. Balancing between exploration and exploitation considerably improves PSO. Introducing an inertia weight, for example, would sort that out.

Clerc and Kennedy (2002) obtain another variation in the speed calculation. A constriction factor $\chi$ is introduced with that purpose, This factor depends on the constants that are used when calculating speed. This factor affects the formula (14). The aim is to avoid the explosion of velocity:

$$
v_{d}^{(i)}=\chi\left[v_{d}^{(i)}+c_{1} \epsilon_{1}\left(p_{d}^{(i)}-x_{d}^{(i)}\right)+c_{2} \epsilon_{2}\left(g_{d}^{(i)}-x_{d}^{(i)}\right)\right]
$$

$$
\chi \text { is: }
$$$$
\chi=\frac{2}{\left|2-\varphi-\sqrt{\varphi^{2}-4 \varphi}\right|}, \quad \varphi=c_{1}+c_{2}=4.1
$$

The results are: $\chi=0.729$ and $c_{1}=c_{2}=2.05$. These parameters were obtained performing several tests.

$\chi$ factor is similar to the inertial weight. This means that controlling the velocity with $V_{(\max )}$ is not required when $\chi$ is used. Bratton and Kennedy (2007), analyzed the stability of this algorithm by using these values and by following a comparative study of both PSO algorithms (inertial weight and $\chi$ factor). Both of them are mathematically equivalent, in particular the algorithm with constriction factor is a special case of the inertial weight. Moreover, Parsopoulos and Vrahatis (2002a) combined both for problems with constraints and they obtained equally good results in several tests.

We observe that the convergence always becomes slower when problem size increase, so when it comes to highdimensional problems, a larger number of iterations occurs. Korenaga et al. (2007) developed a PSO model, where velocity values are updated, by considering the rotation of the coordinate system. This model is aimed at problems of high dimensionality and it showed good results when applied to all functions of Potter and Jong (1994).

\section{Adapting PSO to the MKP}

One of the constraints The MKP problem (6) has is that variables can only be equal to either 0 or 1 . When running PSO, every particle $p$ are set to either 0 or 1 . According to Wang et al. (2008), there are different ways to solve the constraint problem such as the use of penalty functions method, the greedy algorithm or the Surrogate Duality method ( $\mathrm{Li}$ and Sun 2006). When working with constraints $\left\{g_{j}(x)\right\}$, the penalty functions $\varphi$ are the most commonly used. These functions return some degree of solution unfeasibility when the violation of a constraint occurs. 
$\varphi=\sum_{j=1}^{m} \max \left(0, g_{j}(x)\right)$

where the fitness function of a given particle $p_{i}$ is:

fitness $\left(p_{i}\right)=f(x)^{i}+\varphi^{i}$

If there is no constraint violation, then $\varphi^{i}$ is equal to zero. Michalewicz and Schoenauer (1996) show several types of penalty-based algorithms such as static ones, dynamic ones and adaptative ones. Each one of them has advantages and disadvantages.

As this problem involves linear restrictions and binary variables, the most simple method is proposed in this paper which is the one that uses the penalty function (17). The functions output is multiplied by a big constant numberKMAX, and then, the result is evaluated as shown in (18).

In order to solve MKP with PSO this work has been inspired by Hembecker et al. (2007) who used PSO without optimized parameters to deal with the multirestriction problem with a penalty method. Tests were carried out for a sample of data: (Shizuo Senju 1968; Weingartner and Ness 1967; Shih 1979), and the results were fairly closed to the optimal ones. Furthermore, Wan and Nolle (2009) introduced a hybrid algorithm which used a known operator based on heritage (random bits of the "parent" particle gbest y lbest were copied over to the "children" particles). On top of this, they used a mutation operator which updated a bit randomly by using a fix mutation rate equal to 0.33 . The particles then were forced to fulfill all the constraint by deploying a repairing operator. With all these updates, they also reached optimal results for high-dimensional types (Chu and Beasley 1998). A different approach was tried by Wang et al. (2008), who presented a new strategy to update the particle position by using a linear function $L(x)$, with parameters $R_{\min }$ and $R_{\max }$. Wang also used random numbers generation processes to transform a vector of real numbers into a vector of binary values.

\subsection{Proposed algorithm (HPSOGO)}

The proposed implementation, see Algorithm 2, uses PSO for binary variables, although the particles update is performed in three different ways:

(a) An iterative process that uses (12) y (13) to upgrade all the components of the particles. The vector components $\rho_{i}$ are random numbers which are generated in the program.

(b) A random number rand 1 within the interval $(1, M)$ is generated; this value is the number of components to be replaced by the optimal ones (best personal particle) in the current solution (note that $M$ is the dimension of a given particle).

(c) Another random number $\operatorname{rand}_{2}$ within $(1, M)$ is created; this value is the number of components to be replaced by the optimal ones (best particle among the whole swarm) in the current solution.

The update process in item (a) is what is normally used in PSO. Updates items (b) and (c) are similar to the crossover/mutation operations which are commonly used in genetic algorithms. Figure 3 shows operations (b) and (c).

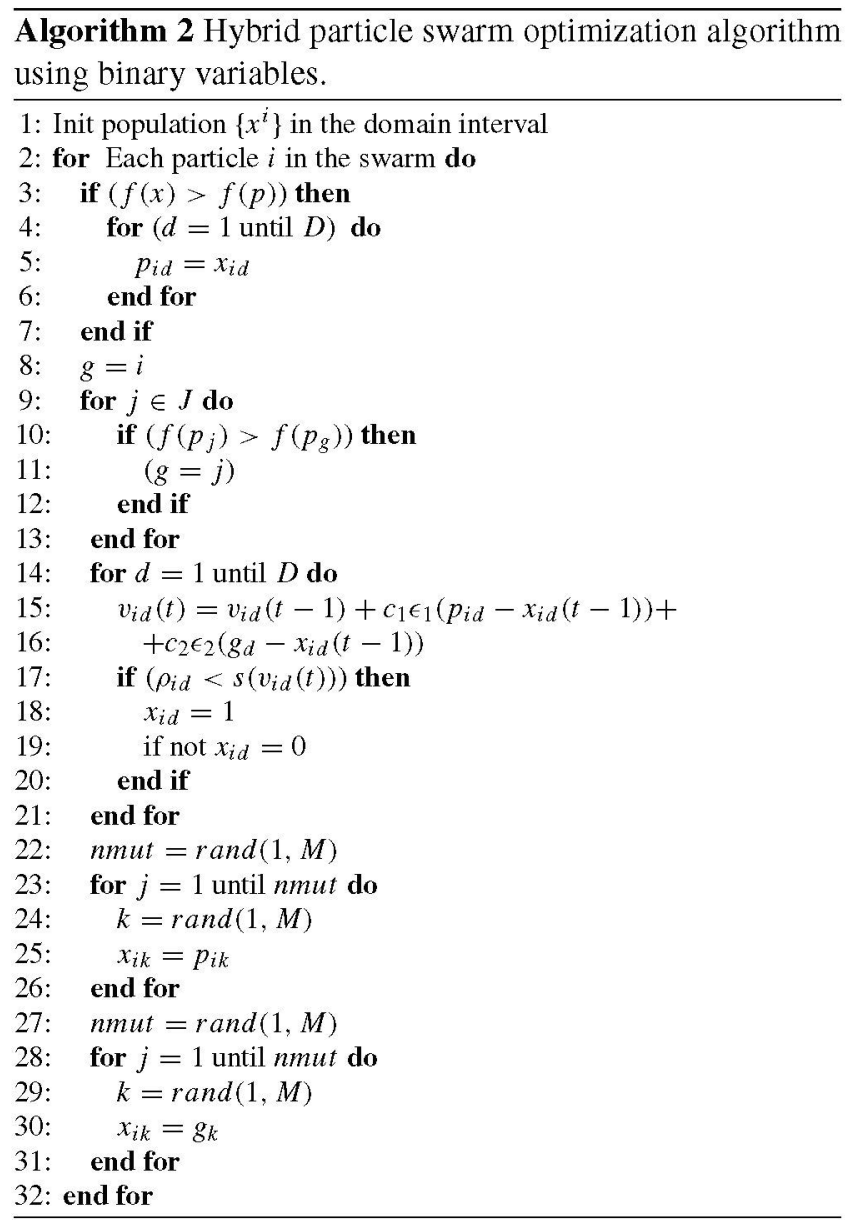

\section{Experiment and results using ORLib benchmarks}

Benchmark data samples were obtained from known problems (Shizuo Senju 1968; Weingartner and Ness 1967; Shih 1979). This section attempts to apply benchmark data sets to model evaluation. Proposed algorithm (HPSOGO) was tested using several groups of MKP benchmarks selected from OR-Library (Beasley 1990). The first group contains 10 
Fig. 3 Genetic mutation over a current particle: 2 random bits from $p_{\text {Best }}$ and 1 random bit from $g_{B}$ est are copied to the current particle

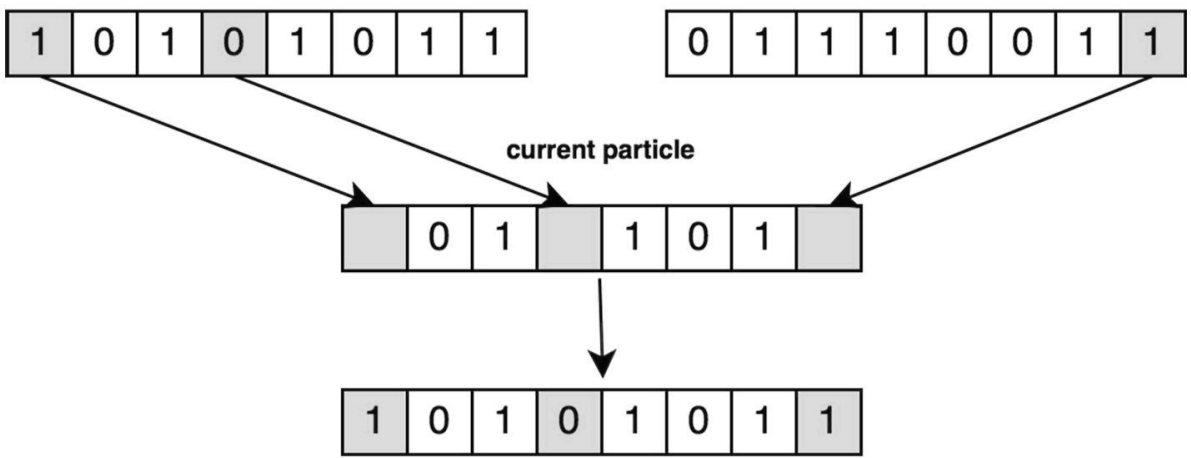

modified current particle
Table 1 Results after applying the algorithm to the Weing data set

\begin{tabular}{lrrrlrl}
\hline Prob. weing & $n$ & $m$ & Optimal & It & Best obtained & gap \\
\hline 1 & 28 & 2 & 141.278 & 360 & 141.278 & 0 \\
2 & 28 & 2 & 130.883 & 360 & 130.883 & 0 \\
3 & 28 & 2 & 95.677 & 2.000 & 95.677 & 0 \\
4 & 28 & 2 & 119.337 & 2.000 & 119.337 & 0 \\
5 & 28 & 2 & 98.796 & 539 & 98.796 & 0 \\
6 & 28 & 2 & 130.623 & 539 & 130.623 & 0 \\
7 & 105 & 2 & 1095.445 & 7.000 & 1094.192 & 0.11 \\
8 & 105 & 2 & 624.319 & 7.000 & 615.927 & 1.34 \\
\hline
\end{tabular}

Table 2 Results after applying the algorithm to the Sento data set

\begin{tabular}{lllllll}
\hline Prob. sento & $n$ & $m$ & Optimal & It & Best obtained & gap \\
\hline 1 & 60 & 30 & 7.772 & 3.605 & 7.772 & 0 \\
2 & 60 & 30 & 8.722 & 5.000 & 8.722 & 0 \\
\hline
\end{tabular}

benchmark problems and corresponds to "SENTO" (Shizuo Senju 1968) and "WEING" (Weingartner and Ness 1967). The second group contains 30 benchmark problems and corresponds to "WEISH" (Shih 1979).

The algorithm was built-in wxDev-C++ and executed 20 times. The swarms had 15 particles. Results are described in Tables 1, 2 and 3. It also shows the number of variables $(n)$, number of constraints $(m)$, the optimal known value, the average number of iterations (it), the optimal value obtained after 20 executions and the gap. Indeed, for the set of the instances tested in this paper the optimal value is already known. This last value is used to measure the error (optimal value known, optimal value found) and it is calculated according to the following expression.

gap $=\frac{\text { optimal value }- \text { best found }}{\text { optimal value }}$
Table 3 Results after applying the algorithm to the Weish data set

\begin{tabular}{lccclcl}
\hline Prob. weish & $n$ & $m$ & Optimal & It & Best obtained & gap \\
\hline 1 & 30 & 5 & 4.554 & 1.000 & 4.554 & 0 \\
2 & 30 & 5 & 4.536 & 1.000 & 4.536 & 0 \\
3 & 30 & 5 & 4.115 & 1.000 & 4.115 & 0 \\
4 & 30 & 5 & 4.561 & 1.000 & 4.561 & 0 \\
5 & 30 & 5 & 4.514 & 500 & 4.514 & 0 \\
6 & 40 & 5 & 5.557 & 1.000 & 5.557 & 0 \\
7 & 40 & 5 & 5.567 & 500 & 5.567 & 0 \\
8 & 40 & 5 & 5.605 & 1.000 & 5.605 & 0 \\
9 & 40 & 5 & 5.246 & 500 & 5.246 & 0 \\
10 & 50 & 5 & 6.339 & 1.000 & 6.339 & 0 \\
11 & 50 & 5 & 5.643 & 2.000 & 5.643 & 0 \\
12 & 50 & 5 & 6.339 & 1.000 & 6.339 & 0 \\
13 & 50 & 5 & 6.159 & 1.000 & 6.159 & 0 \\
14 & 60 & 5 & 6.954 & 1.500 & 6.954 & 0 \\
15 & 60 & 5 & 7.486 & 1.500 & 7.486 & 0 \\
16 & 60 & 5 & 7.289 & 2.000 & 7.289 & 0 \\
17 & 60 & 5 & 8.633 & 2.000 & 8.633 & 0 \\
18 & 70 & 5 & 9.580 & 1.500 & 9.580 & 0 \\
19 & 70 & 5 & 7.698 & 2.000 & 7.698 & 0 \\
20 & 70 & 5 & 9.450 & 4.000 & 9.450 & 0 \\
21 & 70 & 5 & 9.074 & 7.000 & 9.074 & 0 \\
22 & 80 & 5 & 8.947 & 5.400 & 8.947 & 0 \\
23 & 80 & 5 & 8.344 & 5.850 & 8.344 & 0 \\
24 & 80 & 5 & 10.220 & 7.000 & 10.220 & 0 \\
25 & 80 & 5 & 9.939 & 7.000 & 9.939 & 0 \\
26 & 80 & 5 & 9.584 & 8.094 & 9.563 & 0.21 \\
27 & 90 & 5 & 9.819 & 7.197 & 9.819 & 0 \\
28 & 90 & 5 & 9.492 & 7.197 & 9.398 & 0.99 \\
30 & 90 & 5 & 11.191 & 7.000 & 11.191 & 0 \\
\hline & & & & & &
\end{tabular}

If the value is 0 , the optimal solution is found. Note that weing 7 and weing 8 did not obtain the best solution after 20 executions of their algorithm. However in different times, the algorithm was able to find the optimal solutions after 
Table 4 Results after applying the proposed algorithm (HPSOGO) to four problems, in comparison with PBPSO

\begin{tabular}{llllcll}
\hline Prob. & Optimal & Algorithm & $\begin{array}{l}\text { Best } \\
\text { obtained }\end{array}$ & Success rate (\%) & Average fitness & Average execution time \\
\hline Sento1 & 7.772 & PBPSO & 7.772 & 5 & 7.6959 & 3.158 \\
$n=60$ & & HPSOGO & 7.772 & $\mathbf{2 5}$ & $\mathbf{7 . 7 3 3 9}$ & 3.605 \\
$m=30$ & & & & & & \\
Sento2 & 8.722 & PBPSO & 8.772 & 5 & 8.651 & 2.221 \\
$n=60$ & & HPSOGO & 8.722 & $\mathbf{1 0}$ & $\mathbf{8 . 6 8 9 3}$ & 5.000 \\
$m=30$ & & & & & & \\
Weish12 & 6.339 & PBPSO & 6.339 & 45 & 6.33175 & 1.436 \\
$n=50$ & & HPSOGO & 6.339 & 35 & 6.30505 & $\mathbf{1 . 0 0 0}$ \\
$m=5$ & & & & & & \\
Weish20 & 9.450 & PBPSO & 9.450 & 5 & 9.36205 & 3.652 \\
$n=50$ & & HPSOGO & 9.450 & $\mathbf{1 0}$ & $\mathbf{9 . 3 9 8 3 5}$ & 4.000 \\
$m=5$ & & & & & & \\
\hline B.d & & & & & \\
\hline
\end{tabular}

Bold values represent best obtained result
20 executions. This occurred due to the inherent stochastic nature of the technique.

Table 4 shows results using HPSOGO with benchmark problems sento 1, sento2, weish 12 and weish 20 , so it is possible to compare them with the ones obtained by Wang et al. (2008) after executing the probability binary particle swarm optimization algorithm (PBPSO). The entries are: problem's name, optimal known value, applied algorithm, optimal obtained solution, success rate after 20 independent executions, optimal values average after 20 executions and average number of iterations after 20 executions.

After applying the proposed algorithm (HPSOGO) in all the cases, the optimal value was returned (same as with PBPSO). It is noticeable a good performance in the proposed algorithm, although the number of iterations get higher than the ones of PBPSO (mainly because this worked with a 60 particles population). If each particle is evaluated for every iterative step, 2.221 iterations of PBPSO make 133.260 functions be evaluated; when 5.000 iterations of the proposed algorithm with 15 particles are evaluated, 75.000 functions are checked. Furthermore, it showed a better performance than Kennedy and Eberhart algorithms Kennedy and Eberhart (1997), Shen et al. (2004) and Hembecker et al. (2007), for the same particular problems.

\subsection{Comparison with recent research}

These benchmark instances were likewise solved using other existing PSO algorithms: BPSOTVAC (Chih et al. 2014), CBPSOTVAC (Chih et al. 2014), BPSOSIPAC (Lin et al. 2016) and PBPSO (Wang et al. 2008). Previous methods have considered 0-1 MKP benchmark instances where the optimal solution is known. In order to fairly compare the proposed algorithm with that in the previous studies, we mainly followed the measures and parameter settings which are the same as the different existing algorithms used in their original works. Thus, the comparison among these PSO algorithms was performed based on five performance measures used in Chih et al. (2014): success rate (SR) - the value enclosed in parentheses is the quality measure (standard error) of the corresponding estimate-, mean absolute percentage error (MAPE), least error (LE) and standard deviation (SD). SR is the number of runs out of all of the executions that produce the optimum solution within the termination criterion. MAD is the average of the absolute difference between the simulation data and the given optimal solution. MAPE is obtained by dividing MAD by the corresponding optimal solution and usually expresses the accuracy as a percentage. LE is the least error obtained by the minimum of absolute difference between the optimum solution and final solution. SD is the standard deviation of final solutions over runs.

Based on the results of Table 5, the average $85.9 \%$ SR of HPSOGO is superior to the average below $64.8 \% \mathrm{SR}$ of MBPSO, CBPSO1, BPSOTVAC and CBPSOTVAC for the sento and weish. Based on the results, the average $0.4 \% \mathrm{SR}$ of HPSO is superior to the average $0 \%$ SR of BPSOTVAC and CBPSOTVAC. In addition, HPSOGO is superior to MBPSO, CBPSO1, BPSOTVAC and CBPSOTVAC on the measure of MAD, MAPE, LE and SD. Therefore, BPSOSIPAC outperforms the other four PSOs in terms of the four criteria.

HPSOGO was compared with the PSO algorithms for the weing problems. Simulation results are shown in Table 5. Based on the results, the average $100 \%$ SR of HPSOGO is superior to the average below 74.8 SR of BPSO, SCPSO and CBPSOTVAC. In addition, HPSOGO is superior to BPSO, SCPSO and CBPSOTVAC on the measure of SR, MAPE, LE and SD. Therefore, HPSOGO outperforms the other PSOs in terms of the four criteria.

HPSOGO and BPSOSIPAC have a similar performance. 
Table 5 Comparing proposed algorithm with the MBPSO, CBPSO1, BPSOTVAC, CBPSOTVAC and BPSOSIPAC algorithms proposed in Chih et al. (2014)

\begin{tabular}{|c|c|c|c|c|c|c|c|}
\hline Problem & \#Knapsacks & \#Items & Algorithm & SR & MAPE & LE & SD \\
\hline \multirow[t]{6}{*}{ Sento1 } & \multirow[t]{6}{*}{30} & \multirow[t]{6}{*}{60} & MBPSO & $0.16(0.04)$ & $0.0058(0.0006)$ & 229 & 43.23 \\
\hline & & & CBPSO1 & $0(0)$ & $0.026(0.001)$ & 302 & 49.92 \\
\hline & & & BPSOTVAC & $0.57(0.05)$ & $0.0011(0.0001)$ & 34 & 11.52 \\
\hline & & & CBPSOTVAC & $0.39(0.05)$ & $0.021(0.007)$ & 3146 & 357.78 \\
\hline & & & BPSOSIPAC & $1(0)$ & $0(0)$ & 0 & 0 \\
\hline & & & HPSOGO & $0.25(0.01)$ & $0(0)$ & 35 & 9.21 \\
\hline \multirow[t]{6}{*}{ Sento2 } & \multirow[t]{6}{*}{30} & \multirow[t]{6}{*}{60} & MBPSO & $0.03(0.02)$ & $0.0029(0.0002)$ & 81 & 18.8 \\
\hline & & & CBPSO 1 & $0(0)$ & $0.012(0.0003)$ & 150 & 25.78 \\
\hline & & & BPSOTVAC & $0.27(0.04)$ & $0.001(0.0001)$ & 38 & 7.04 \\
\hline & & & CBPSOTVAC & $0.2(0.04)$ & $0.0063(0.0012)$ & 633 & 101.03 \\
\hline & & & BPSOSIPAC & $1(0)$ & $0(0)$ & 0 & 0 \\
\hline & & & HPSOGO & $0.1(0.02)$ & $0(0)$ & 52 & 8.01 \\
\hline \multirow[t]{6}{*}{ Weing1 } & \multirow[t]{6}{*}{2} & \multirow[t]{6}{*}{28} & MBPSO & $0.82(0.04)$ & $0.0008(0.0002)$ & 801 & 250.43 \\
\hline & & & CBPSO1 & $1(0)$ & $0(0)$ & 0 & 0 \\
\hline & & & BPSOTVAC & $1(0)$ & $0(0)$ & 0 & 0 \\
\hline & & & CBPSOTVAC & $0.92(0.03)$ & $0.0004(0.0002)$ & 1961 & 281.98 \\
\hline & & & BPSOSIPAC & $1(0)$ & $0(0)$ & 0 & 0 \\
\hline & & & HPSOGO & $1(0)$ & $0(0)$ & 0 & 0 \\
\hline \multirow[t]{6}{*}{ Weing2 } & \multirow[t]{6}{*}{2} & \multirow[t]{6}{*}{28} & MBPSO & $0.65(0.05)$ & $0.0009(0.0002)$ & 1700 & 314.08 \\
\hline & & & CBPSO1 & $1(0)$ & $0(0)$ & 0 & 0 \\
\hline & & & BPSOTVAC & $1(0)$ & $0(0)$ & 0 & 0 \\
\hline & & & CBPSOTVAC & $0.88(0.03)$ & $0.0009(0.0004)$ & 3341 & 545.5 \\
\hline & & & BPSOSIPAC & $1(0)$ & $0(0)$ & 0 & 0 \\
\hline & & & HPSOGO & $1(0)$ & $0(0)$ & 0 & 0 \\
\hline \multirow[t]{6}{*}{ Weing3 } & \multirow[t]{6}{*}{2} & \multirow[t]{6}{*}{28} & MBPSO & $0.11(0.03)$ & $0.0112(0.0009)$ & 3500 & 876.78 \\
\hline & & & CBPSO1 & $1(0)$ & $0(0)$ & 0 & 0 \\
\hline & & & BPSOTVAC & $0.92(0.03)$ & $0.00007(0.00003)$ & 160 & 25.53 \\
\hline & & & CBPSOTVAC & $0.75(0.04)$ & $0.0019(0.0007)$ & 3789 & 672.42 \\
\hline & & & BPSOSIPAC & $1(0)$ & $0(0)$ & 0 & 0 \\
\hline & & & HPSOGO & $1(0)$ & $0(0)$ & 0 & 0 \\
\hline \multirow[t]{6}{*}{ Weing4 } & \multirow[t]{6}{*}{2} & \multirow[t]{6}{*}{28} & MBPSO & $0.76(0.04)$ & $0.0049(0.0011)$ & 4001 & 1270.8 \\
\hline & & & CBPSO1 & $1(0)$ & $0(0)$ & 0 & 0 \\
\hline & & & BPSOTVAC & $1(0)$ & $0(0)$ & 0 & 0 \\
\hline & & & CBPSOTVAC & $0.97(0.02)$ & $0.0004(0.0003)$ & 3774 & 378.58 \\
\hline & & & BPSOSIPAC & $1(0)$ & $0(0)$ & 0 & 0 \\
\hline & & & HPSOGO & $1(0)$ & $0(0)$ & 0 & 0 \\
\hline \multirow[t]{6}{*}{ Weing 5} & \multirow[t]{6}{*}{2} & \multirow[t]{6}{*}{28} & MBPSO & $0.52(0.05)$ & $0.017(0.002)$ & 4778 & 1923.5 \\
\hline & & & CBPSO1 & $1(0)$ & $0(0)$ & 0 & 0 \\
\hline & & & BPSOTVAC & $1(0)$ & $0(0)$ & 0 & 0 \\
\hline & & & CBPSOTVAC & $0.94(0.02)$ & $0.0009(0.0006)$ & 4728 & 572.82 \\
\hline & & & BPSOSIPAC & $1(0)$ & $0(0)$ & 0 & 0 \\
\hline & & & HPSOGO & $1(0)$ & $0(0)$ & 0 & 0 \\
\hline Weing6 & 2 & 28 & MBPSO & $0.36(0.05)$ & $0.0023(0.0002)$ & 1340 & 322.4 \\
\hline & & & CBPSO1 & $1(0)$ & $0(0)$ & 0 & 0 \\
\hline & & & BPSOTVAC & $0.97(0.02)$ & $0.00009(0.00005)$ & 390 & 66.86 \\
\hline & & & CBPSOTVAC & $0.87(0.03)$ & $0.0007(0.0003)$ & 2460 & 343.45 \\
\hline & & & BPSOSIPAC & $1(0)$ & $0(0)$ & 0 & 0 \\
\hline & & & HPSOGO & $1(0)$ & $0(0)$ & 0 & 0 \\
\hline
\end{tabular}




\begin{tabular}{|c|c|c|c|c|c|c|c|}
\hline Problem & \#Knapsacks & \#Items & Algorithm & SR & MAPE & LE & SD \\
\hline \multirow[t]{6}{*}{ Weing7 } & \multirow[t]{6}{*}{2} & \multirow[t]{6}{*}{105} & MBPSO & $0.02(0.01)$ & $0.0006(0.0001)$ & 6111 & 1130.6 \\
\hline & & & CBPSO1 & $0(0)$ & $0.0308(0.0005)$ & 42,425 & 5002 \\
\hline & & & BPSOTVAC & $0(0)$ & $0.00026(0.00004)$ & 2069 & 383.74 \\
\hline & & & CBPSOTVAC & $0(0)$ & $0.011(0.003)$ & 154,486 & 30,020 \\
\hline & & & BPSOSIPAC & $1(0)$ & $0(0)$ & $\mathbf{0}$ & 0 \\
\hline & & & HPSOGO & $1(0)$ & $0.11(0.3)$ & 0 & 0 \\
\hline \multirow[t]{6}{*}{ Weing8 } & \multirow[t]{6}{*}{2} & \multirow[t]{6}{*}{105} & MBPSO & $0.03(0.02)$ & $0.0095(0.0008)$ & 44,731 & 4704.3 \\
\hline & & & CBPSO1 & $0(0)$ & $0.234(0.004)$ & 160,402 & 15,988 \\
\hline & & & BPSOTVAC & $0.35(0.05)$ & $0.0030(0.0003)$ & 6463 & 2000.9 \\
\hline & & & CBPSOTVAC & $0.20(0.04)$ & $13.7(13.6)$ & 623,862 & 75,169 \\
\hline & & & BPSOSIPAC & $1(0)$ & $0(0)$ & $\mathbf{0}$ & 0 \\
\hline & & & HPSOGO & $1(0)$ & $1.34(1.36)$ & $\mathbf{0}$ & 0 \\
\hline \multirow[t]{6}{*}{ Weish1 } & \multirow[t]{6}{*}{5} & \multirow[t]{6}{*}{30} & MBPSO & $0.82(0.04)$ & $0.0024(0.0006)$ & 114 & 26.34 \\
\hline & & & CBPSO1 & $1(0)$ & $0(0)$ & 0 & 0 \\
\hline & & & BPSOTVAC & $1(0)$ & $0(0)$ & 0 & 0 \\
\hline & & & CBPSOTVAC & $0.94(0.02)$ & $0.0012(0.0008)$ & 248 & 32.81 \\
\hline & & & BPSOSIPAC & $1(0)$ & $0(0)$ & 0 & 0 \\
\hline & & & HPSOGO & $1(0)$ & $0(0)$ & 0 & 0 \\
\hline \multirow[t]{6}{*}{ Weish2 } & \multirow[t]{6}{*}{5} & \multirow[t]{6}{*}{30} & MBPSO & $0.55(0.05)$ & $0.0018(0.0004)$ & 123 & 18.01 \\
\hline & & & CBPSO1 & $0.79(0.04)$ & $0.00023(0.00005)$ & 5 & 2.04 \\
\hline & & & BPSOTVAC & $0.64(0.05)$ & $0.00040(0.00005)$ & 5 & 2.41 \\
\hline & & & CBPSOTVAC & $0.66(0.05)$ & $0.0009(0.0005)$ & 231 & 23.12 \\
\hline & & & BPSOSIPAC & $1(0)$ & $0(0)$ & 0 & 0 \\
\hline & & & HPSOGO & $1(0)$ & $0(0)$ & 0 & 0 \\
\hline \multirow[t]{6}{*}{ Weish3 } & \multirow[t]{6}{*}{5} & \multirow[t]{6}{*}{30} & MBPSO & $0.63(0.05)$ & $0.0051(0.0009)$ & 141 & 34.98 \\
\hline & & & CBPSO1 & $1(0)$ & $0(0)$ & 0 & 0 \\
\hline & & & BPSOTVAC & $0.99(0.01)$ & $0.00015(0.00015)$ & 63 & 6.3 \\
\hline & & & CBPSOTVAC & $0.95(0.02)$ & $0.0024(0.0014)$ & 394 & 52.69 \\
\hline & & & BPSOSIPAC & $1(0)$ & $0(0)$ & 0 & 0 \\
\hline & & & HPSOGO & $1(0)$ & $0(0)$ & 0 & 0 \\
\hline \multirow[t]{6}{*}{ Weish4 } & \multirow[t]{6}{*}{5} & \multirow[t]{6}{*}{30} & MBPSO & $0.96(0.02)$ & $0.0004(0.0002)$ & 56 & 8.99 \\
\hline & & & CBPSO1 & $1(0)$ & $0(0)$ & 0 & 0 \\
\hline & & & BPSOTVAC & $1(0)$ & $0(0)$ & 0 & 0 \\
\hline & & & CBPSOTVAC & $0.99(0.01)$ & $0.0023(0.0023)$ & 859 & 85.9 \\
\hline & & & BPSOSIPAC & $1(0)$ & $0(0)$ & 0 & 0 \\
\hline & & & HPSOGO & $1(0)$ & $0(0)$ & 0 & 0 \\
\hline \multirow[t]{6}{*}{ Weish5 } & \multirow[t]{6}{*}{5} & \multirow[t]{6}{*}{30} & MBPSO & $0.99(0.01)$ & $0.00012(0.00012)$ & 54 & 5.4 \\
\hline & & & CBPSO1 & $1(0)$ & $0(0)$ & 0 & 0 \\
\hline & & & BPSOTVAC & $1(0)$ & $0(0)$ & 0 & 0 \\
\hline & & & CBPSOTVAC & $0.98(0.01)$ & $0.0021(0.0019)$ & 742 & 74.45 \\
\hline & & & BPSOSIPAC & $1(0)$ & $0(0)$ & 0 & 0 \\
\hline & & & HPSOGO & $1(0)$ & $0(0)$ & 0 & 0 \\
\hline Weish6 & 5 & 40 & MBPSO & $0.32(0.05)$ & $0.0028(0.0003)$ & 56 & 14.39 \\
\hline & & & CBPSO1 & $0.65(0.05)$ & $0.00098(0.00014)$ & 34 & 7.92 \\
\hline & & & BPSOTVAC & $0.59(0.05)$ & $0.00121(0.00015)$ & 18 & 8.19 \\
\hline & & & CBPSOTVAC & $0.53(0.05)$ & $0.0044(0.0016)$ & 518 & 79.28 \\
\hline & & & BPSOSIPAC & $1(0)$ & $0(0)$ & 0 & 0 \\
\hline & & & HPSOGO & $1(0)$ & $0(0)$ & 0 & 0 \\
\hline
\end{tabular}




\begin{tabular}{|c|c|c|c|c|c|c|c|}
\hline Problem & \#Knapsacks & \#Items & Algorithm & SR & MAPE & LE & SD \\
\hline \multirow[t]{6}{*}{ Weish7 } & \multirow[t]{6}{*}{5} & \multirow[t]{6}{*}{40} & MBPSO & $0.64(0.05)$ & $0.0018(0.0003)$ & 122 & 18.92 \\
\hline & & & CBPSO1 & $0.83(0.04)$ & $0.0006(0.0001)$ & 25 & 7.79 \\
\hline & & & BPSOTVAC & $0.96(0.02)$ & $0.00013(0.00006)$ & 18 & 3.45 \\
\hline & & & CBPSOTVAC & $0.78(0.04)$ & $0.0036(0.0014)$ & 511 & 71.95 \\
\hline & & & BPSOSIPAC & $1(0)$ & $0(0)$ & 0 & 0 \\
\hline & & & HPSOGO & $1(0)$ & $0(0)$ & 0 & 0 \\
\hline \multirow[t]{6}{*}{ Weish8 } & \multirow[t]{6}{*}{5} & \multirow[t]{6}{*}{40} & MBPSO & $0.44(0.05)$ & $0.0013(0.0002)$ & 72 & 13.07 \\
\hline & & & CBPSO 1 & $0.64(0.05)$ & $0.00013(0.00002)$ & 2 & 0.96 \\
\hline & & & BPSOTVAC & $0.79(0.04)$ & $0.00008(0.00001)$ & 2 & 0.82 \\
\hline & & & CBPSOTVAC & $0.68(0.05)$ & $0.0016(0.0008)$ & 418 & 42.81 \\
\hline & & & BPSOSIPAC & $1(0)$ & $0(0)$ & 0 & 0 \\
\hline & & & HPSOGO & $1(0)$ & $0(0)$ & 0 & 0 \\
\hline \multirow[t]{6}{*}{ Weish9 } & \multirow[t]{6}{*}{5} & \multirow[t]{6}{*}{40} & MBPSO & $0.78(0.04)$ & $0.0021(0.0005)$ & 200 & 25.65 \\
\hline & & & CBPSO1 & $0.96(0.02)$ & $0.0003(0.0001)$ & 34 & 6.69 \\
\hline & & & BPSOTVAC & $1(0)$ & $0(0)$ & 0 & 0 \\
\hline & & & CBPSOTVAC & $0.85(0.04)$ & $0.0027(0.0014)$ & 641 & 65.7 \\
\hline & & & BPSOSIPAC & $1(0)$ & $0(0)$ & 0 & 0 \\
\hline & & & HPSOGO & $1(0)$ & $0(0)$ & 0 & 0 \\
\hline \multirow[t]{6}{*}{ Weish10 } & \multirow[t]{6}{*}{5} & \multirow[t]{6}{*}{50} & MBPSO & $0.56(0.05)$ & $0.0017(0.0004)$ & 83 & 22.17 \\
\hline & & & CBPSO1 & $0.07(0.03)$ & $0.0068(0.0005)$ & 141 & 33.65 \\
\hline & & & BPSOTVAC & $0.91(0.03)$ & $0.0002(0.0002)$ & 68 & 9.56 \\
\hline & & & CBPSOTVAC & $0.67(0.05)$ & $0.0102(0.0037)$ & 1394 & 188.63 \\
\hline & & & BPSOSIPAC & $1(0)$ & $0(0)$ & 0 & 0 \\
\hline & & & HPSOGO & $1(0)$ & $0(0)$ & 0 & 0 \\
\hline \multirow[t]{6}{*}{ Weish11 } & \multirow[t]{6}{*}{5} & \multirow[t]{6}{*}{50} & MBPSO & $0.4(0.05)$ & $0.0053(0.0008)$ & 167 & 43.95 \\
\hline & & & CBPSO1 & $0.05(0.02)$ & $0.0144(0.0008)$ & 191 & 47.06 \\
\hline & & & BPSOTVAC & $0.88(0.03)$ & $0.0013(0.0005)$ & 113 & 25.72 \\
\hline & & & CBPSOTVAC & $0.62(0.05)$ & $0.028(0.011)$ & 2245 & 403.03 \\
\hline & & & BPSOSIPAC & $1(0)$ & $0(0)$ & 0 & 0 \\
\hline & & & HPSOGO & $1(0)$ & $0(0)$ & 0 & 0 \\
\hline \multirow[t]{6}{*}{ Weish12 } & \multirow[t]{6}{*}{5} & \multirow[t]{6}{*}{50} & MBPSO & $0.65(0.05)$ & $0.0026(0.0006)$ & 226 & 35.68 \\
\hline & & & CBPSO1 & $0.09(0.03)$ & $0.0092(0.0007)$ & 191 & 42.35 \\
\hline & & & BPSOTVAC & $0.89(0.03)$ & $0.00005(0.00003)$ & 19 & 1.91 \\
\hline & & & CBPSOTVAC & $0.71(0.04)$ & $0.020(0.006)$ & 1497 & 304.43 \\
\hline & & & BPSOSIPAC & $1(0)$ & $0(0)$ & 0 & 0 \\
\hline & & & HPSOGO & $0.35(0.01)$ & $0(0)$ & 34 & 3.83 \\
\hline \multirow[t]{6}{*}{ Weish13 } & \multirow[t]{6}{*}{5} & \multirow[t]{6}{*}{50} & MBPSO & $0.87(0.03)$ & $0.0014(0.0004)$ & 155 & 25.19 \\
\hline & & & CBPSO 1 & $0.15(0.04)$ & $0.0098(0.0007)$ & 159 & 40.97 \\
\hline & & & BPSOTVAC & $1(0)$ & $0(0)$ & 0 & 0 \\
\hline & & & CBPSOTVAC & $0.85(0.04)$ & $0.0075(0.0039)$ & 1725 & 180.04 \\
\hline & & & BPSOSIPAC & $1(0)$ & $0(0)$ & 0 & 0 \\
\hline & & & HPSOGO & $1(0)$ & $0(0)$ & 0 & 0 \\
\hline Weish14 & 5 & 60 & MBPSO & $0.66(0.05)$ & $0.0023(0.0004)$ & 100 & 25.95 \\
\hline & & & CBPSO1 & $0(0)$ & $0.031(0.001)$ & 347 & 66.79 \\
\hline & & & BPSOTVAC & $0.98(0.01)$ & $0.00089(0.00006)$ & 31 & 4.36 \\
\hline & & & CBPSOTVAC & $0.79(0.04)$ & $0.021(0.007)$ & 2127 & 364.66 \\
\hline & & & BPSOSIPAC & $1(0)$ & $0(0)$ & 0 & 0 \\
\hline & & & HPSOGO & $1(0)$ & $0(0)$ & 0 & 0 \\
\hline
\end{tabular}




\begin{tabular}{|c|c|c|c|c|c|c|c|}
\hline Problem & \#Knapsacks & \#Items & Algorithm & SR & MAPE & LE & SD \\
\hline \multirow[t]{6}{*}{ Weish15 } & \multirow[t]{6}{*}{5} & \multirow[t]{6}{*}{60} & MBPSO & $0.72(0.05)$ & $0.0014(0.0002)$ & 70 & 18.64 \\
\hline & & & CBPSO1 & $0(0)$ & $0.0266(0.0008)$ & 359 & 59.99 \\
\hline & & & BPSOTVAC & $1(0)$ & $0(0)$ & 0 & 0 \\
\hline & & & CBPSOTVAC & $0.8(0.04)$ & $0.030(0.011)$ & 2978 & 554.35 \\
\hline & & & BPSOSIPAC & $1(0)$ & $0(0)$ & 0 & 0 \\
\hline & & & HPSOGO & $1(0)$ & $0(0)$ & 0 & 0 \\
\hline \multirow[t]{6}{*}{ Weish16 } & \multirow[t]{6}{*}{5} & \multirow[t]{6}{*}{60} & MBPSO & $0.44(0.05)$ & $0.0011(0.0002)$ & 87 & 17.49 \\
\hline & & & CBPSO 1 & $0(0)$ & $0.0195(0.0007)$ & 259 & 47.07 \\
\hline & & & BPSOTVAC & $0.54(0.05)$ & $0.00016(0.00002)$ & 8 & 1.71 \\
\hline & & & CBPSOTVAC & $0.43(0.05)$ & $0.023(0.006)$ & 1931 & 367.29 \\
\hline & & & BPSOSIPAC & $1(0)$ & $0(0)$ & 0 & 0 \\
\hline & & & HPSOGO & $1(0)$ & $0(0)$ & 0 & 0 \\
\hline \multirow[t]{6}{*}{ Weish17 } & \multirow[t]{6}{*}{5} & \multirow[t]{6}{*}{60} & MBPSO & $0.56(0.05)$ & $0.0007(0.0001)$ & 41 & 7.38 \\
\hline & & & CBPSO1 & $0(0)$ & $0.0107(0.0004)$ & 170 & 32.79 \\
\hline & & & BPSOTVAC & $1(0)$ & $0(0)$ & 0 & 0 \\
\hline & & & CBPSOTVAC & $0.72(0.05)$ & $0.011(0.003)$ & 1035 & 227.16 \\
\hline & & & BPSOSIPAC & $1(0)$ & $0(0)$ & 0 & 0 \\
\hline & & & HPSOGO & $1(0)$ & $0(0)$ & 0 & 0 \\
\hline \multirow[t]{6}{*}{ Weish18 } & \multirow[t]{6}{*}{5} & \multirow[t]{6}{*}{70} & MBPSO & $0.38(0.05)$ & $0.0015(0.0002)$ & 94 & 18.4 \\
\hline & & & CBPSO1 & $0(0)$ & $0.0278(0.0006)$ & 367 & 54.01 \\
\hline & & & BPSOTVAC & $0.75(0.04)$ & $0.00029(0.00005)$ & 15 & 5.25 \\
\hline & & & CBPSOTVAC & $0.53(0.05)$ & $0.011(0.003)$ & 1595 & 275.53 \\
\hline & & & BPSOSIPAC & $1(0)$ & $0(0)$ & 0 & 0 \\
\hline & & & HPSOGO & $1(0)$ & $0(0)$ & 0 & 0 \\
\hline \multirow[t]{6}{*}{ Weish19 } & \multirow[t]{6}{*}{5} & \multirow[t]{6}{*}{70} & MBPSO & $0.55(0.05)$ & $0.0027(0.0004)$ & 149 & 33.67 \\
\hline & & & CBPSO1 & $0(0)$ & $0.059(0.001)$ & 615 & 83.65 \\
\hline & & & BPSOTVAC & $0.65(0.05)$ & $0.0006(0.0001)$ & 35 & 7.13 \\
\hline & & & CBPSOTVAC & $0.62(0.05)$ & $0.028(0.009)$ & 3060 & 489.37 \\
\hline & & & BPSOSIPAC & $1(0)$ & $0(0)$ & 0 & 0 \\
\hline & & & HPSOGO & $1(0)$ & $0(0)$ & 0 & 0 \\
\hline \multirow[t]{6}{*}{ Weish20 } & \multirow[t]{6}{*}{5} & \multirow[t]{6}{*}{70} & MBPSO & $0.53(0.05)$ & $0.0011(0.0002)$ & 69 & 15.99 \\
\hline & & & CBPSO1 & $0(0)$ & $0.037(0.001)$ & 528 & 89.41 \\
\hline & & & BPSOTVAC & $0.78(0.04)$ & $0.0004(0.00008)$ & 20 & 7.53 \\
\hline & & & CBPSOTVAC & $0.69(0.05)$ & $0.015(0.005)$ & 2482 & 410.74 \\
\hline & & & BPSOSIPAC & $1(0)$ & $0(0)$ & 0 & 0 \\
\hline & & & HPSOGO & $0.1(0.01)$ & $0(0)$ & 52 & 15.01 \\
\hline \multirow[t]{6}{*}{ Weish21 } & \multirow[t]{6}{*}{5} & \multirow[t]{6}{*}{70} & MBPSO & $0.61(0.05)$ & $0.0019(0.0003)$ & 88 & 24.97 \\
\hline & & & CBPSO 1 & $0(0)$ & $0.039(0.001)$ & 499 & 84.42 \\
\hline & & & BPSOTVAC & $0.74(0.04)$ & $0.0007(0.0001)$ & 24 & 10.41 \\
\hline & & & CBPSOTVAC & $0.67(0.05)$ & $0.016(0.005)$ & 2574 & 378.38 \\
\hline & & & BPSOSIPAC & $1(0)$ & $0(0)$ & 0 & 0 \\
\hline & & & HPSOGO & $1(0)$ & $0(0)$ & 0 & 0 \\
\hline Weish22 & 5 & 80 & MBPSO & $0.33(0.05)$ & $0.0033(0.0004)$ & 112 & 31.55 \\
\hline & & & CBPSO1 & $0(0)$ & $0.082(0.001)$ & 935 & 94.69 \\
\hline & & & BPSOTVAC & $0.16(0.04)$ & $0.00169(0.00007)$ & 18 & 6.63 \\
\hline & & & CBPSOTVAC & $0.17(0.04)$ & $0.024(0.007)$ & 3063 & 486.71 \\
\hline & & & BPSOSIPAC & $1(0)$ & $0(0)$ & 0 & 0 \\
\hline & & & HPSOGO & $1(0)$ & $0(0)$ & 0 & 0 \\
\hline
\end{tabular}


Table 5 continued

\begin{tabular}{|c|c|c|c|c|c|c|c|}
\hline Problem & \#Knapsacks & \#Items & Algorithm & SR & MAPE & LE & SD \\
\hline \multirow[t]{6}{*}{ Weish23 } & 5 & 80 & MBPSO & $0.24(0.04)$ & $0.0036(0.0004)$ & 126 & 35.43 \\
\hline & & & CBPSO 1 & $0(0)$ & $0.087(0.002)$ & 902 & 119.64 \\
\hline & & & BPSOTVAC & $0.85(0.04)$ & $0.00013(0.00006)$ & 36 & 5.11 \\
\hline & & & CBPSOTVAC & $0.58(0.05)$ & $0.026(0.007)$ & 3114 & 437.23 \\
\hline & & & BPSOSIPAC & $1(0)$ & $0(0)$ & 0 & 0 \\
\hline & & & HPSOGO & $1(0)$ & $0(0)$ & 0 & 0 \\
\hline \multirow[t]{6}{*}{ Weish24 } & 5 & 80 & MBPSO & $0.27(0.04)$ & $0.0017(0.0002)$ & 70 & 18.09 \\
\hline & & & CBPSO 1 & $0(0)$ & $0.0373(0.0006)$ & 499 & 56.96 \\
\hline & & & BPSOTVAC & $0.7(0.05)$ & $0.00029(0.00006)$ & 31 & 6.44 \\
\hline & & & CBPSOTVAC & $0.55(0.05)$ & $0.012(0.003)$ & 1841 & 295.79 \\
\hline & & & BPSOSIPAC & $1(0)$ & $0(0)$ & 0 & 0 \\
\hline & & & HPSOGO & $1(0)$ & $0(0)$ & 0 & 0 \\
\hline \multirow[t]{6}{*}{ Weish 25} & 5 & 80 & MBPSO & $0.29(0.05)$ & $0.0015(0.0001)$ & 61 & 13.39 \\
\hline & & & CBPSO1 & $0(0)$ & $0.0475(0.0009)$ & 648 & 86.21 \\
\hline & & & BPSOTVAC & $0.49(0.05)$ & $0.00045(0.00007)$ & 24 & 7.09 \\
\hline & & & CBPSOTVAC & $0.32(0.05)$ & $0.013(0.004)$ & 2321 & 361.88 \\
\hline & & & BPSOSIPAC & $1(0)$ & $0(0)$ & 0 & 0 \\
\hline & & & HPSOGO & $1(0)$ & $0(0)$ & 0 & 0 \\
\hline \multirow[t]{6}{*}{ Weish26 } & 5 & 90 & MBPSO & $0.31(0.05)$ & $0.0028(0.0002)$ & 114 & 24.27 \\
\hline & & & CBPSO1 & $0(0)$ & $0.103(0.001)$ & 1122 & 126.39 \\
\hline & & & BPSOTVAC & $0.36(0.05)$ & $0.0012(0.0001)$ & 47 & 12.81 \\
\hline & & & CBPSOTVAC & $0.28(0.04)$ & $0.04(0.01)$ & 4084 & 710.77 \\
\hline & & & BPSOSIPAC & $1(0)$ & $0(0)$ & 0 & 0 \\
\hline & & & HPSOGO & $1(0)$ & $0.21(0.0005)$ & 0 & 0 \\
\hline \multirow[t]{6}{*}{ Weish27 } & 5 & 90 & MBPSO & $0.65(0.05)$ & $0.0024(0.0005)$ & 203 & 48.62 \\
\hline & & & CBPSO1 & $0(0)$ & $0.109(0.001)$ & 1205 & 120.34 \\
\hline & & & BPSOTVAC & $0.99(0.01)$ & $0.00004(0.00004)$ & 39 & 3.9 \\
\hline & & & CBPSOTVAC & $0.83(0.04)$ & $0.028(0.009)$ & 3915 & 640.43 \\
\hline & & & BPSOSIPAC & $1(0)$ & $0(0)$ & 0 & 0 \\
\hline & & & HPSOGO & $1(0)$ & $0(0)$ & 0 & 0 \\
\hline \multirow[t]{6}{*}{ Weish28 } & 5 & 90 & MBPSO & $0.64(0.05)$ & $0.0016(0.0003)$ & 144 & 26.72 \\
\hline & & & CBPSO1 & $0(0)$ & $0.115(0.002)$ & 1266 & 122.32 \\
\hline & & & BPSOTVAC & $0.87(0.03)$ & $0.00031(0.00008)$ & 23 & 7.77 \\
\hline & & & CBPSOTVAC & $0.62(0.05)$ & $0.06(0.02)$ & 4387 & 887.33 \\
\hline & & & BPSOSIPAC & $1(0)$ & $0(0)$ & 0 & 0 \\
\hline & & & HPSOGO & $1(0)$ & $0.99(0.43)$ & 0 & 0 \\
\hline \multirow[t]{6}{*}{ Weish30 } & 5 & 90 & MBPSO & $0.38(0.05)$ & $0.001(0.0001)$ & 57 & 14.48 \\
\hline & & & CBPSO1 & $0(0)$ & $0.0516(0.0009)$ & 760 & 87.58 \\
\hline & & & BPSOTVAC & $0.87(0.03)$ & $0.00005(0.00001)$ & 4 & 1.35 \\
\hline & & & CBPSOTVAC & $0.63(0.05)$ & $0.021(0.005)$ & 2836 & 491.81 \\
\hline & & & BPSOSIPAC & $1(0)$ & $0(0)$ & 0 & 0 \\
\hline & & & HPSOGO & $1(0)$ & $0(0)$ & 0 & 0 \\
\hline
\end{tabular}

Bold values represent best obtained result 


\section{Conclusion}

This paper proposes a new binary algorithm, HPSOGO, to solve the multidimensional knapsack problem. This algorithm has proven to be effective in terms of performance in comparison with other PSO-based algorithms. The proposed algorithm finds the optimal solution by evaluating the functions a smaller number of times. All benchmarks are based on multidimensional constraints. Furthermore, it a very manageable algorithm, easy to implement, adaptable to discrete structures and it does not require parameters' adjustment.

In this study, a novel PSO algorithm, named HPSOGO, has been used to solve the MKPs. Several benchmark problems (Sento, Weing, Weish) from OR-Library were tested, and the results were compared with other existing PSO algorithms. Benchmarks used in HPSOGO are based on the multidimensional constraints.

The major conclusion is that this binary algorithm has proven to be effective in terms of performance in comparison with the other PSO-based algorithms. Our proposal finds the optimal solution by evaluating the functions a fairly small number of times. Furthermore, it is a very manageable algorithm, easy to implement, adaptable to discrete structures and it does not require parameters' adjustment.

Although the proposed algorithm works very well for MKP, further research needs to be conducted when dealing with other problems not MKP related before speaking about universality and robustness, the proposal must be tested and evaluated in other binary problems. In addition, the algorithm should increase the success rate in high-dimensional knapsack problems. Finally, comparing with the existing non-PSO state-of-the-art methods is helpful in establishing the algorithm for general purposes.

Acknowledgements The research was partially supported by Spanish Research Agency Projects TRA2013-48314-C3-2-R and SEGVAUTO TRIES (S2013/MIT-27139).

\section{Compliance with ethical standards}

Conflict of interest The authors declare that they have no conflict of interest.

Ethical approval This article does not contain any studies with human participants or animals performed by any of the authors.

\section{References}

Beasley J (1990) Or-library: distributing test problems by electronic mail. J Oper Res Soc 41(11):1069-1072

Bratton D, Kennedy J (2007) Defining a standard for particle swarm optimization. In: 2007 IEEE Swarm Intelligence Symposium, SIS 2007, Honolulu, Hawaii, USA, 1-5 April 2007, pp 120-127
Chih M, Lin CJ, Chern MS, Ou TY (2014) Particle swarm optimization with time-varying acceleration coefficients for the multidimensional knapsack problem. Appl Math Model 38(4):1338-1350. doi:10.1016/j.apm.2013.08.009

Chu P, Beasley J (1998) A genetic algorithm for the multidimensional knapsack problem. J Heuristics 4(1):63-86. doi:10.1023/ A: 1009642405419

Clerc M, Kennedy J (2002) The particle swarm-explosion, stability, and convergence in a multidimensional complex space. Trans Evol Comput 6(1):58-73

Eberhart R, Kennedy J (1995) A new optimizer using particle swarm theory. In: Proceedings of the sixth international symposium on micro machine and human science, 1995. MHS '95. pp 39-43. doi:10.1109/MHS.1995.494215

Haddar B, Khemakhem M, Hanafi S, Wilbaut C (2016) A hybrid quantum particle swarm optimization for the multidimensional knapsack problem. Eng Appl Artif Intell 55:1-13. doi:10.1016/ j.engappai.2016.05.006

Haddar B, Khemakhem M, Rhimi H, Chabchoub H (2016b) A quantum particle swarm optimization for the $0-1$ generalized knapsack sharing problem. Nat Comput 15(1):153-164. doi:10.1007/ s11047-014-9470-5

Hembecker F, Lopes H, Godoy W (2007) Particle swarm optimization for the multidimensional knapsack problem. Springer, Berlin

Kellerer H, Pferschy U, Pisinger D (2004) Knapsack problems. Springer, Berlin

Kennedy J, Eberhart R (1997) A discrete binary version of the particle swarm algorithm. In: Proceedings of the IEEE international conference on systems, man, and cybernetics, IEEE computer society, Washington, DC, USA, vol 5. pp 4104-4108

Kennedy J, Eberhart RC (1995) Particle swarm optimization. In: Proceedings of the IEEE international conference on neural networks. pp 1942-1948

Kennedy J, Mendes R (2006) Neighborhood topologies in fully informed and best-of-neighborhood particle swarms. IEEE Trans Syst Man Cybern C (Appl Rev) 36(4):515-519. doi:10.1109/ TSMCC. 2006.875410

Korenaga T, Hatanaka T, Uosaki K (2007) Performance improvement of particle swarm optimization for high-dimensional function optimization. In: 2007 IEEE congress on evolutionary computation. pp 3288-3293

Korte B, Vygen J (2007) Combinatorial optimization: theory and algorithms, 4th edn. Springer Publishing Company, Incorporated, Berlin

Ktari R, Chabchoub H (2013) Essential particle swarm optimization queen with tabu search for MKP resolution. Computing 95(9):897921. doi: 10.1007/s00607-013-0316-2

Labed S, Gherboudj A, Chikhi S (2011) Article: a modified hybrid particle swarm optimization algorithm for multidimensional knapsack problem. Int J Comput Appl 34(2):11-16 (full text available)

Li D, Sun X (eds) (2006) Surrogate duality theory. Springer, Boston

Lin CJ, Chern MS, Chih M (2016) A binary particle swarm optimization based on the surrogate information with proportional acceleration coefficients for the $0-1$ multidimensional knapsack problem. J Ind Prod Eng 33(2):77-102. doi:10.1080/21681015.2015.1111263

Martello S, Toth P(1985) Algorithm 632: a program for the 0-1 multiple knapsack problem. ACM Trans Math Softw 11(2):135-140

Martello S, Toth P (1990) Knapsack problems: algorithms and computer implementations. Wiley, New York

Michalewicz Z, Schoenauer M (1996) Evolutionary algorithms for constrained parameter optimization problems. Evol Comput 4(1): 1-32

Parsopoulos K, Vrahatis M (2002) Particle swarm optimization method for constrained optimization problems. In: Proceedings of the Euro-international symposium on computational intelligence 2002. IOS Press, pp 214-220 
Parsopoulos K, Vrahatis M (2002b) Recent approaches to global optimization problems through particle swarm optimization. Nat Comput Int J 1(2-3):235-306

Potter M, Jong KD (1994) A cooperative coevolutionary approach to function optimization. In: Proceedings of the international conference on evolutionary computation. The third conference on parallel problem solving from nature: parallel problem solving from nature. Springer, London, UK, PPSN III, pp 249-257

Shen Q, Jiang JH, Jiao CX, li Shen G, Yu RQ (2004) Modified particle swarm optimization algorithm for variable selection in MLR and PLS modeling: QSAR studies of antagonism of angiotensin II antagonists. Eur J Pharm Sci 22(2-3):145-152

Shi Y, Eberhart R (1998) A modified particle swarm optimizer. In: Proceedings of IEEE international conference on evolutionary computation. IEEE Computer Society, Washington, DC, USA, pp $69-73$

Shih W (1979) A branch and bound method for the multiconstraint zeroone knapsack problem. J Oper Res Soc 30(4):369-378. doi:10. $1057 /$ jors. 1979.78
Shizuo S, Toyoda Y (1968) An approach to linear programming with 0-1 variables. Manag Sci 15(4):B196-B207

Wan NF, Nolle L (2009) Solving a multi-dimensional knapsack problem using a hybrid particle swarm optimization algorithm. In: ECMS. pp 93-98

Wang L, Wang X, Fu J, Zhen L (2008) A novel probability binary particle swarm optimization algorithm and its application. JSW 3(9):28-35

Weingartner M, Ness D (1967) Methods for the solution of multidimensional 0/1 knapsack problems. Oper Res 15:83-103

Zhang L, Malik S (2002) The quest for efficient Boolean satisfiability solvers. In: Proceedings of the 14th international conference on computer aided verification. Springer, London, UK, CAV '02, pp $17-36$ 